\title{
Tumor infiltrating neutrophil might play a major role in predicting the clinical outcome of breast cancer patients treated with neoadjuvant chemotherapy
}

\author{
Sheng-Kai Geng ${ }^{1 \dagger}$, Shao-Mei Fu ${ }^{2 \dagger}$, Shi-Hong Ma ${ }^{1}$, Yi-Peng Fu ${ }^{2^{*}}$ and Hong-Wei Zhang ${ }^{3^{*}}$
}

\begin{abstract}
Background: This study was aimed to explore the predictive ability of tumor infiltrating neutrophil (TIN) in patients with breast cancer treated with neoadjuvant chemotherapy (NACT). Furthermore, the significance of TIN's dynamic change before and after NACT was investigated.

Methods: Between January 2004 and December 2017, a total of 133 patients with breast cancer who underwent NACT before surgery were enrolled in this retrospective cohort. Eighty-nine of them were able to get the core needle biopsy (CNB) samples and all the pathological samples after surgery were available. TIN was detected by immunohistochemical staining of CD66b. The optimal cut-off value was determined via receiver operating characteristic (ROC) curve analysis. The association of clinicopathologic characteristics and chemotherapy efficiency was analyzed using $X^{2}$ test or Fisher's exact test or t-test as appropriate, and the prognostic significances were assessed by univariate and multivariate analyses.

Results: Patients with higher TIN after NACT were confirmed to be significantly associated with worse prognosis $(P=0.002)$. After stratifying patients into two groups, high difference group was prone to have better chemotherapy efficiency $(P<0.001)$ and clinical outcome in both univariate $(P=0.002)$ and multivariate analyses $(P=0.003)$.

Conclusions: In this study, higher TIN after NACT was confirmed to be associated with breast cancer patients' worse chemotherapy efficiency and shorter disease-free survival (DFS). Furthermore, the TIN's dynamic change before and after NACT was firstly proved to be a more accurate predictive marker compared with TIN after NACT.
\end{abstract}

Keywords: Breast cancer, Tumor infiltrating neutrophil, Chemotherapy efficiency, Disease-free survival, Neoadjuvant chemotherapy

\footnotetext{
*Correspondence: fyp0076@163.com; zhang.hongwei@zs-hospital.sh.cn

†Sheng-Kai Geng and Shao-Mei Fu contributed equally to this work.

${ }^{2}$ Department of Breast Surgeon, The Obstetrics \& Gynecology Hospital of Fudan University, Shanghai 200011, China

${ }^{3}$ Department of General Surgery, Zhongshan Hospital, Fudan University, Shanghai 200032, China

Full list of author information is available at the end of the article
}

(c) The Author(s). 2021 Open Access This article is licensed under a Creative Commons Attribution 4.0 International License, which permits use, sharing, adaptation, distribution and reproduction in any medium or format, as long as you give appropriate credit to the original author(s) and the source, provide a link to the Creative Commons licence, and indicate if changes were made. The images or other third party material in this article are included in the article's Creative Commons licence, unless indicated otherwise in a credit line to the material. If material is not included in the article's Creative Commons licence and your intended use is not permitted by statutory regulation or exceeds the permitted use, you will need to obtain permission directly from the copyright holder. To view a copy of this licence, visit http://creativecommons.org/licenses/by/4.0/ The Creative Commons Public Domain Dedication waiver (http://creativecommons.org/publicdomain/zero/1.0/) applies to the data made available in this article, unless otherwise stated in a credit line to the data. 


\section{Background}

Breast cancer is the leading cause of cancer-related deaths in women worldwide [1]. With the enhancement of health awareness and improvement of detection equipment, the incidence of breast cancer has significantly increased in young women [2]. Due to the aggressive biological characteristics [3] and aesthetic concerns, neoadjuvant chemotherapy (NACT) has been widely accepted by breast cancer patients to achieve breastconserving (BCS) and cosmetic surgery. Although some patients could benefit from NACT, easily accessible and reliable clinical markers are needed to stratify the prognosis of this population.

Immune response plays an essential role in chemotherapy-mediated tumor eradication, which is reflected by the tumor infiltration immune cells in the tumor microenvironment (TME) [4-7], such as tumor infiltrating neutrophils (TIN), which have emerged as activators and regulators of tumor immunity in TME [812]. For instance, Galdiero et al. [13] revealed that colorectal cancer patients who had higher TIN density showed better response to 5-FU-based chemotherapy. While in other researches, higher TIN was identified as a prognostic marker for poor clinical outcome in some malignant tumors such as renal cell carcinoma [14], and head and squamous cell carcinoma [15]. Wang, Y et al. also observed that neutrophils in tumor parenchyma were an independent factor for poor prognosis in breast cancer patients [16]. The present study revealed that neutrophils induced breast cancer epithelialmesenchymal transition (EMT) via tissue inhibitor of metalloproteinase-1 (TIMP-1). Reciprocally, breast cancer cells undergoing EMT enhanced neutrophil TIMP-1 secretion by CD90 in a cell-contact manner. Shaul, ME et al. hypothesized that tumor neutrophils play an important role in the recruitment of immunocytes, and in maintaining the balance between the activation and suppression of the immune system in cancer [17]. However, TIN has been rarely reported in breast cancer, and the significance of dynamic changes in TIN before and after NACT in TME remains unknown.

The goal of this study was to evaluate the clinical value of TIN in patients with breast cancer treated with NACT. Furthermore, the significance of dynamic change in TIN before and after NACT was investigated.

\section{Methods}

\section{Patients}

A total of 133 patients with breast cancer who underwent NACT before surgery were enrolled in this study, of which 89 underwent core needle biopsy (CNB) before NACT, and all the pathological samples after surgery were available. This study was approved by the Institutional Review Board of Zhongshan Hospital, Fudan
University (B2020-415). The patients were enrolled from the Zhongshan Hospital, Fudan University, between January 2004 and December 2017. The inclusion and exclusion criteria were as follows: all patients were pathologically diagnosed with breast cancer; all patients received radical treatment for breast cancer; all the blood samples were collected within 3 days before operations; all patients underwent NACT before surgery; chemotherapy efficacy of all patients was evaluated after NACT based on response evaluation criteria in solid tumors (RECIST) combined with pathological residual tumor cells; postoperative pathological samples of all patients were stained to count CD66b positive TINs using immunohistochemistry. We used one marker to identify TIN because after careful search, CD66b neutrophils were found to be localized either within the blood vessels or diffusely scattered throughout the tumor. Jensen HK et al. used double staining (CD34 and CD66b) for neutrophils mainly because there may be more vascular tissue in renal cell carcinoma [18], while other studies only used CD66b staining to represent neutrophils because they comprise $96 \%$ of the granulocyte population [19-21]. In our study, breast cancer specimens were mainly gland tissue; 89 patients' $\mathrm{CNB}$ samples before NACT were stained to count CD66b positive TINs using immunohistochemistry; all patients had complete records and follow-up data, including baseline characteristics such as sex, age, menopause status, stage, molecular type, and preoperative routine blood test.

\section{Histopathological evaluation}

Chemotherapy efficacy of all patients was evaluated after NACT based on RECIST [22] combined with pathological evaluation of residual tumor cells. Patients were stratified into two groups, remission group and no remission group. The remission group included patients who achieved complete response $(\mathrm{CR})$ and partial response (PR). For pathological evaluation, CR was defined as the complete absence of invasive disease in the breast tumor according to the (NSABP) B-18 protocol [23], and $\mathrm{PR}$ was defined as the absence of invasive tumor or only focal residual invasive carcinoma cells in the primary site [24]. The no remission group included patients who achieved stable disease (SD) and progressive disease (PD) [22].

Immunohistochemistry and evaluation of immunostaining The immunohistochemical protocols were performed as previously described [19, 20, 25, 26]. We briefly described as follows: Formalin-fixed paraffin-embedded surgical specimens were used for the immunohistochemical analysis. The presence of available tumor was confirmed by hematoxylin and eosin staining. The tissue blocks were sectioned at $2 \mu \mathrm{m}$ and mounted on glass 
slides. The sections were dewaxed in xylene and graded alcohols, hydrated, and washed in phosphate-buffered saline (PBS). After the endogenous peroxidase was inhibited using 3\% $\mathrm{H} 2 \mathrm{O} 2$ for $30 \mathrm{~min}$, the sections were pretreated in a microwave oven ( $15 \mathrm{~min}$ in sodium citrate buffer, $\mathrm{pH} 6$ ) and then incubated with $10 \%$ normal goat serum for $30 \mathrm{~min}$. The mouse antihuman monoclonal anti-CD66b primary antibody (clone G10F5, diluted at 1 : 200, BD Biosciences) was applied at room temperature for $1.5 \mathrm{~h}$, and washed with PBS. Then, the biotinylatedlabeled secondary antibody was applied for $30 \mathrm{~min}$ followed by streptavidin peroxidase. Finally, the sections were stained with hematoxylin for $1 \mathrm{~min}$ and mounted with an aqueous mounting medium supplied with the kit. Digital pathological section scanner system was used to scan and collect high-definition staining images. To count the number of tumor-infiltrating immune cells, we randomly selected five high-power fields of view (HPF, 200X), and calculated the average as the count. Positive staining was generally brown and located in the cell membrane. The value of TIN pre-NACT, TIN postNACT and the change of TIN before and after NACT were calculated (the value of TIN's change equals the number of pre-NACT TIN minus the number of postNACT TIN). According to the optimal cut-off value of TIN's change, patients were stratified into the high difference group and the low difference group. The evaluation of CD66b immunostaining was independently conducted by two pathologists who were blinded to the clinical data, and the results were averaged.

\section{Follow-up}

All patients underwent postoperative follow-up every 3 months during the first postoperative year, and every 6 months thereafter. Routine blood test, chest X-ray, tumor markers, breast, liver, gallbladder, pancreas and spleen ultrasonography were performed in every followup. Bone-scan was performed every 12 months. The disease free survival (DFS) was defined as the interval between surgery and time of recurrence for relapsed patients or from the date of surgery to the date of last follow-up for nonrecurrent patients.

\section{Statistical analysis}

Statistical analysis was performed using SPSS version 22 . The optimal cut-off value of TIN's change was determined via receiver operating characteristic curve (ROC) curve analysis. Youden index is the sum of the sensitivity and specificity minus 1 . We used Youden index to find the optimal cut-off value. The association between clinicopathological characteristics and patients' clinical outcome was analyzed using $\mathrm{X}^{2}$ test or Fisher's exact test. The Kaplan-Meier analysis was used to compare the survival differences of patients between different cohorts.
The Cox proportional hazards regression model was used to perform univariate and multivariate analyses. For all statistical tests, $p<0.05$ (two-tailed) was considered significant.

\section{Results}

\section{Clinicopathological profiles of the patients}

The clinicopathological characteristics of the patients treated with NACT are presented in Table 1. The median follow-up time was 27 months (range from 10 to 109 months). The median age of all patients was $54.15 \pm$

Table 1 Clinicopathological profiles of the patients treated with NACT

\begin{tabular}{|c|c|c|c|}
\hline \multicolumn{4}{|c|}{ Characteristic } \\
\hline \multicolumn{2}{|c|}{ Age } & \multicolumn{2}{|c|}{$54.15 \pm 10.747$} \\
\hline \multicolumn{2}{|c|}{$<40$} & 14 & $10.5 \%$ \\
\hline \multicolumn{2}{|c|}{$>40$} & 119 & $89.5 \%$ \\
\hline \multicolumn{2}{|c|}{ No menopause } & 49 & $36.8 \%$ \\
\hline \multicolumn{2}{|c|}{ Menopause } & 84 & $63.2 \%$ \\
\hline \multicolumn{2}{|l|}{ T1 } & 20 & $15.0 \%$ \\
\hline \multicolumn{2}{|l|}{ T2 } & 80 & $60.2 \%$ \\
\hline \multicolumn{2}{|l|}{ T3 } & 26 & $19.5 \%$ \\
\hline \multicolumn{2}{|l|}{ T4 } & 7 & $5.3 \%$ \\
\hline \multicolumn{2}{|l|}{ No } & 36 & $27.1 \%$ \\
\hline \multicolumn{2}{|l|}{ N1 } & 27 & $20.3 \%$ \\
\hline \multicolumn{2}{|l|}{ N2 } & 32 & $24.1 \%$ \\
\hline \multicolumn{2}{|l|}{ N3 } & 38 & $28.6 \%$ \\
\hline \multicolumn{2}{|c|}{ Stage I } & 9 & $6.8 \%$ \\
\hline \multicolumn{2}{|c|}{ Stage II } & 46 & $34.6 \%$ \\
\hline \multicolumn{2}{|c|}{ Stage III } & 78 & $58.6 \%$ \\
\hline \multicolumn{2}{|c|}{$\mathrm{HR}+$} & 88 & $66.2 \%$ \\
\hline \multicolumn{2}{|l|}{ HR- } & 45 & $33.8 \%$ \\
\hline \multicolumn{2}{|c|}{ Her-2 positive } & 17 & $12.8 \%$ \\
\hline \multicolumn{2}{|c|}{ Her-2 negative } & 116 & $87.2 \%$ \\
\hline \multicolumn{2}{|c|}{ TNBC vs } & 28 & $21.1 \%$ \\
\hline \multicolumn{2}{|c|}{ No TNBC } & 105 & $78.9 \%$ \\
\hline \multicolumn{2}{|c|}{ Recurrence VS } & 24 & $18.0 \%$ \\
\hline \multicolumn{2}{|c|}{ No recurrence } & 109 & $82.9 \%$ \\
\hline \multirow[t]{4}{*}{ CR } & TEC & 71 & $53.4 \%$ \\
\hline & EC & 23 & $17.3 \%$ \\
\hline & $\mathrm{TC}$ & 12 & $9.0 \%$ \\
\hline & TX & 27 & $20.3 \%$ \\
\hline \multicolumn{2}{|c|}{ Post-NACT neutrophils } & \multicolumn{2}{|c|}{$2.92 \pm 1.72$} \\
\hline \multicolumn{2}{|c|}{ Post-NACT lymphocyte } & \multicolumn{2}{|c|}{$1.30 \pm 0.48$} \\
\hline
\end{tabular}

Abbreviation: $H R$ Hormone receptor, Her-2 Human epidermal growth factor receptor-2, TNBC Triple negative breast cancer, NACT Neoadjuvant chemotherapy, CR Chemical regime, TEC Docetaxel + anthracyclines + cyclophosphamide, EC Anthracyclines + cyclophosphamide, $T C$ Docetaxel + cyclophosphamide, $T X$ Docetaxel + platinum 
10.747 years. Meanwhile, 24 of 133 breast cancer patients experienced recurrences during follow-up. Among all breast cancer patients, 88 were hormone receptor positive $(\mathrm{HR}+$ ) patients (39 Luminal A patients and 49 Luminal B patients), 17 were human epidermal receptor positive $(\mathrm{Her}-2+)$ patients and 28 were triple negative breast cancer (TNBC) patients. In terms of the chemical regime of patients treated with $\mathrm{NACT}, 71$ patients were treated with docetaxel + anthracyclines + cyclophosphamide (TEC) plans, 23 patients were treated with anthracyclines + cyclophosphamide (EC) plans, 12 were treated with docetaxel + cyclophosphamide (TC) plans, and 27 patients were treated with docetaxel + platinum (DP) plans.

\section{Cut-off value of TIN pre-NACT, TIN post-NACT and its dynamic change}

CD66b positive staining varied in different specimens of breast cancer patients, and was diffusely scattered throughout the tumor. The value of CD66b positive TIN ranged from 0.2 to 225 (typical staining is shown in Supplement Fig. 3), the typical staining of TIN's dynamic change is shown in Supplement Fig. 4.

In this study, there was no optimal cut-off value of the TIN before NACT for chemotherapy efficacy, and the area under the ROC curve was 0.449 (95\% confidence interval $[\mathrm{CI}], 0.275-0.622, p=0.473$, shown in Fig. 1a). The optimal cut-off value of TIN after NACT for resistance to chemotherapy was 52.2, and the area under ROC curve was 0.643 (95\% [CI], 0.509-0.776, $p=0.019$, shown in Fig. 1b). The optimal cut-off value of the TIN's (CD66b positive staining) change associated with the strongest Youden index for the chemotherapy efficacy was 5.75. The area under the ROC curve was 0.770 (95\% [CI], 0.657-0.883, $p<0.001$, showed in Fig. 1c).
The value of TIN for chemotherapy efficacy and DFS

Although there was no optimal cut-off value of TIN before NACT for chemotherapy efficacy, the optimal cutoff value of TIN after NACT for resistance to chemotherapy was 52.2. After stratifying patients into two groups, the clinicopathological characteristics of each group are listed in Table 2. Patients with higher TIN were more likely to suffer from resistance after treatment with NACT $(p<0.001$, shown in Supplement Fig. 2a). In addition, after long term follow-up, higher TIN after NACT was proved to be associated with significantly shorter DFS $(p=0.002$, hazard ratio $[\mathrm{HR}]=$ $3.415 ; 95 \%[\mathrm{CI}] 1.489-7.831$ in univariate analysis, shown in Fig. 2a, and $p=0.007$, hazard ratio $[\mathrm{HR}]=$ 3.265 ; $95 \%$ [CI $] 1.374-7.758$ in multivariate analysis, shown in Table 3). The prognostic value of TIN' change was analyzed as follows.

\section{Relationship between the value of TIN's change and patient characteristics}

After confirming the optimal cut-off value of the TIN's change, 89 breast cancer patients whose pathological specimens were available before and after NACT were classified into two groups, the high difference group $(n=48)$ and the low difference group $(n=41)$. The clinicopathological characteristics of each group are listed in Table 4. There were no significant differences between the value of TIN's change and menopause status, tumor size, lymph node metastasis, stage and chemical regimes $(p=0.495$, shown in Supplement Fig. 1).

\section{The value of TIN's change before and after NACT for chemotherapy efficacy and DFS in patients with breast cancer}

In this study, low difference value of TIN was associated with high rates of recurrence and low rates of remission

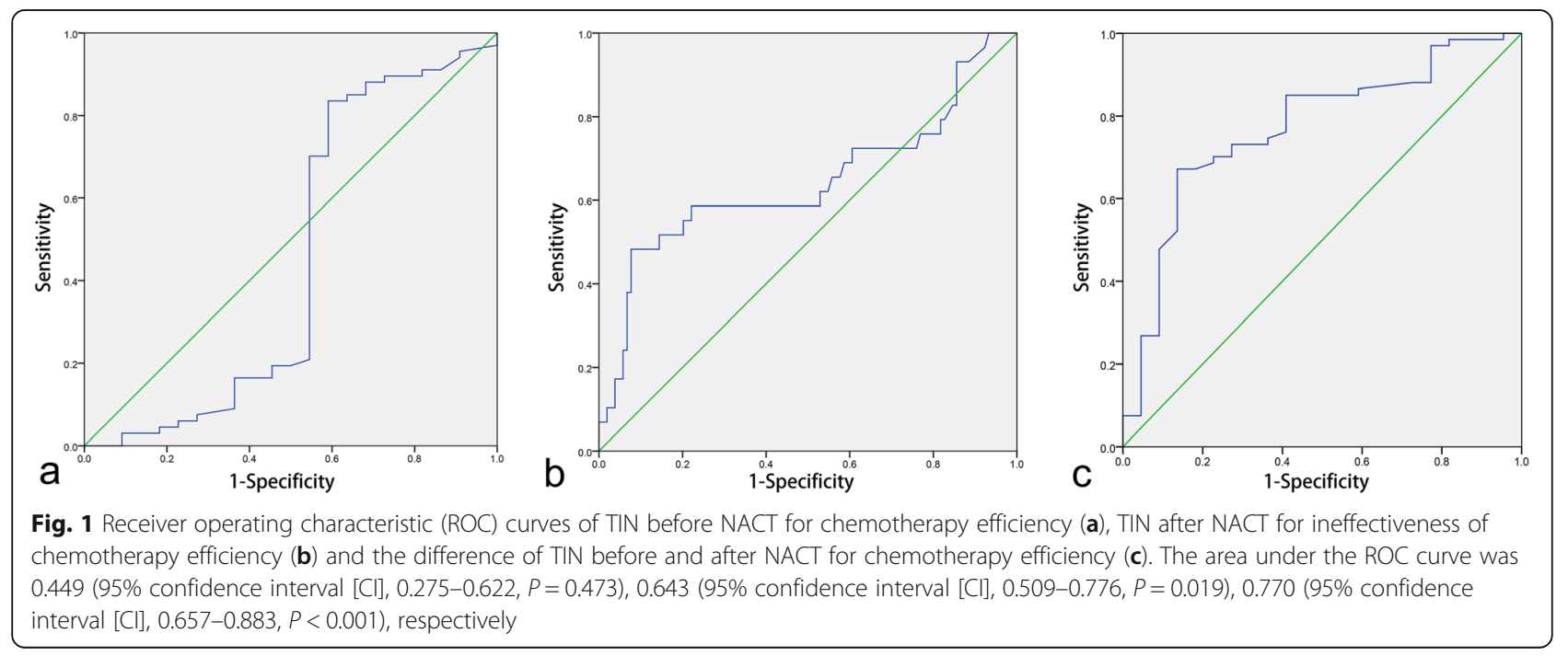


Table 2 Clinicopathological profiles of the patients treated with NACT (stratified by TIN after NACT)

\begin{tabular}{|c|c|c|c|c|}
\hline \multirow{2}{*}{\multicolumn{2}{|c|}{ Characteristic }} & Low TIN after NACT & High TIN after NACT & $P$ \\
\hline & & $N=111$ & $N=22$ & \\
\hline \multicolumn{2}{|c|}{ Age } & $54.02 \pm 10.872$ & $54.82 \pm 10.312$ & 0.804 \\
\hline \multicolumn{2}{|c|}{$<40$} & 12 & 2 & 0.810 \\
\hline \multicolumn{2}{|c|}{$>40$} & 99 & 20 & \\
\hline \multicolumn{2}{|c|}{ No menopause } & 42 & 7 & 0.593 \\
\hline \multicolumn{2}{|c|}{ Menopause } & 69 & 15 & \\
\hline \multicolumn{2}{|l|}{$\mathrm{T} 1$} & 17 & 3 & 0.791 \\
\hline \multicolumn{2}{|l|}{$\mathrm{T} 2$} & 68 & 12 & \\
\hline \multicolumn{2}{|l|}{ T3 } & 21 & 5 & \\
\hline \multicolumn{2}{|l|}{ T4 } & 5 & 2 & \\
\hline \multicolumn{2}{|l|}{ No } & 33 & 3 & 0.382 \\
\hline \multicolumn{2}{|l|}{ N1 } & 21 & 6 & \\
\hline \multicolumn{2}{|l|}{ N2 } & 25 & 7 & \\
\hline \multicolumn{2}{|l|}{ N3 } & 32 & 6 & \\
\hline \multicolumn{2}{|c|}{ Stage I } & 9 & 0 & 0.214 \\
\hline \multicolumn{2}{|c|}{ Stage II } & 40 & 6 & \\
\hline \multicolumn{2}{|c|}{ Stage III } & 62 & 16 & \\
\hline \multicolumn{2}{|c|}{$\mathrm{HR}+$} & 77 & 11 & 0.079 \\
\hline \multicolumn{2}{|c|}{ HR- } & 34 & 11 & \\
\hline \multicolumn{2}{|c|}{ Her-2 positive } & 11 & 6 & 0.026 \\
\hline \multicolumn{2}{|c|}{ Her-2 negative } & 100 & 16 & \\
\hline \multicolumn{2}{|c|}{ TNBC vs } & 23 & 5 & 0.833 \\
\hline \multicolumn{2}{|c|}{ No TNBC } & 88 & 17 & \\
\hline \multicolumn{2}{|c|}{ Remission vs } & 96 & 8 & $<0.001$ \\
\hline \multicolumn{2}{|c|}{ No remission } & 15 & 14 & \\
\hline \multicolumn{2}{|c|}{ Recurrence vs } & 15 & 9 & 0.002 \\
\hline \multicolumn{2}{|c|}{ No recurrence } & 96 & 13 & \\
\hline \multirow[t]{4}{*}{ CR } & TEC & 60 & 11 & 0.089 \\
\hline & $\mathrm{EC}$ & 20 & 3 & \\
\hline & $\mathrm{EC}$ & 9 & 3 & \\
\hline & TX & 22 & 5 & \\
\hline
\end{tabular}

Abbreviation: HR Hormone receptor, Her-2 Human epidermal growth factor receptor-2, TNBC Triple negative breast cancer, NACT Neoadjuvant chemotherapy, CR Chemical regime, TEC Docetaxel + anthracyclines + cyclophosphamide, EC Anthracyclines + cyclophosphamide, TC Docetaxel + cyclophosphamide, DP Docetaxel + platinum

(shown in Fig. 2b and Supplement Fig. 2, b). In Supplement Table 1, the value of TIN's change was identified as a significant predictor for DFS ( $p=0.002$, hazard ratio $[\mathrm{HR}]=0.984 ; 95 \%[\mathrm{CI}]$ 0.974-0.994 in univariate analysis). In multivariate analysis, the value of TIN's change was significantly associated with the rate of recurrence $(p=0.003$, hazard ratio $[\mathrm{HR}]=0.984 ; 95 \%[\mathrm{CI}] 0.973-$ 0.994), and the C-index of TIN's change $(0.64,95 \% \mathrm{CI}$ 0.63-0.65) was higher than that of TIN after NACT (0.62, 95\% [CI] 0.61-0.63).

\section{Discussion}

In this study, patients with higher TIN after NACT were more inclined to suffer resistance to NACT and recurrence. In addition, this is the first study to demonstrate that the TIN's dynamic change before and after NACT was significantly associated with better chemotherapy efficacy and prognosis in patients with breast cancer.

Previous researches have confirmed the prognostic role of tumor infiltrating lymphocyte (TIL) in breast cancer patients [27-31]. As an indispensable component 


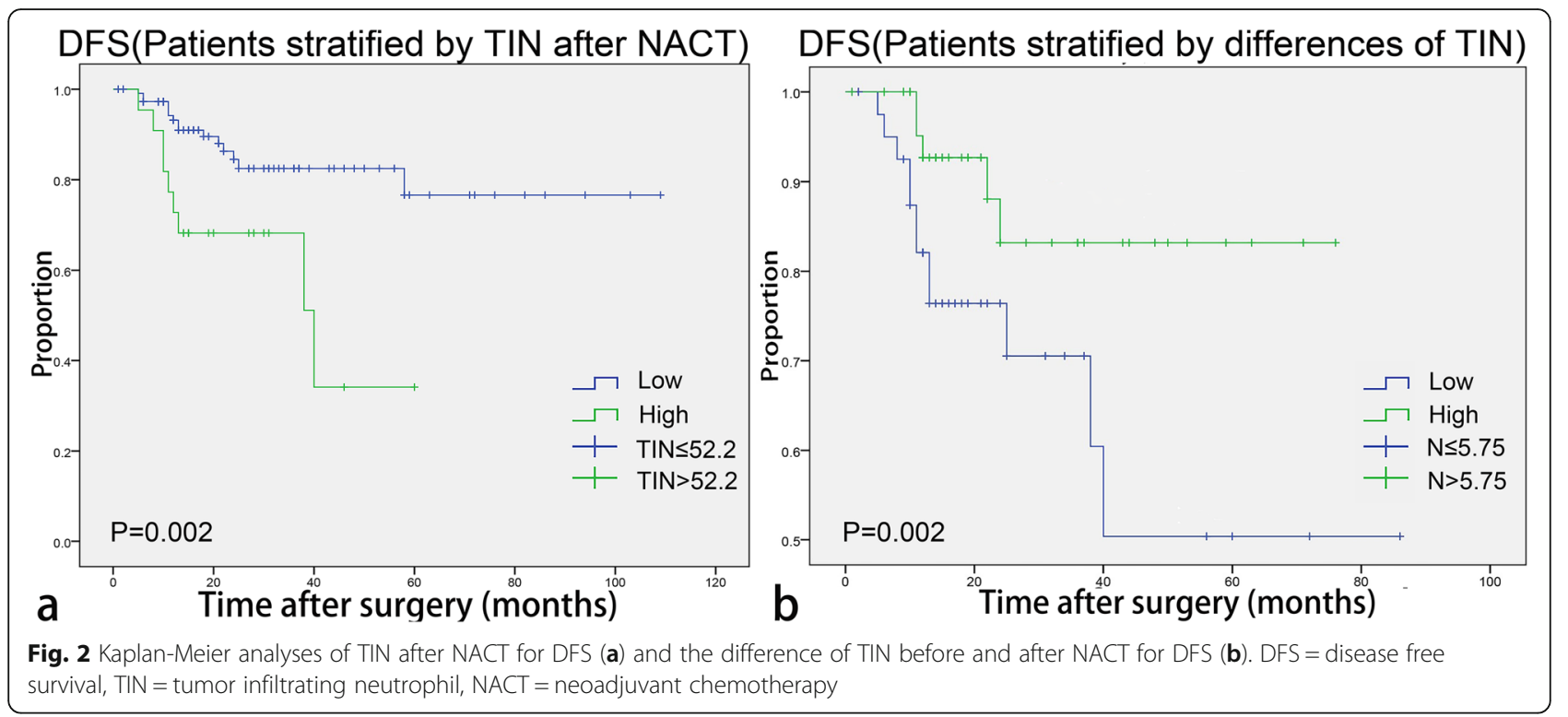

of tumor immune cells, TIN was reported to have a significant impact on TME [32]. Wang J et al. [33] had implicated TIN as an independent marker of prognosis in biliary cancer patients. Jensen HK et al. [18] revealed that presence of TIN was an independent prognostic factor in localized renal cell carcinoma. When concerning breast cancer, studies have revealed that TIN may be associated with metastasis [16, 34]. After stratifying patients into two groups according to the optimal cut-off value of TIN after NACT for chemotherapy efficacy, in accordance with previous studies, the subgroup with lower TIN $(<52.2)$ was associated with longer DFS. In addition, patients with higher TIN after NACT were more inclined to be resistant to NACT. The reason may be that the higher concentration of TIN after NACT correlates with the inhibition of anti-tumor immunity and the initiation of metastasis [16, 34]. However, the specific mechanism accounting for the association remains unclear.
The change of composition in TME may reflect the TME's response to NACT. Given that the status of TIN after NACT could be associated with clinical outcome of breast cancer patients, we concentrated further on the significance of dynamic change of TIN before and after NACT. In this study, we revealed that it was significantly associated with chemotherapy efficacy (shown in Table $2, p<0.001$ ) and prognosis of breast cancer patients $(p=0.002$, hazard ratio $[\mathrm{HR}]=$ 0.984; $95 \%$ [CI] $0.974-0.994$ in univariate analysis and $p=$ 0.003 , hazard ratio $[\mathrm{HR}]=0.984 ; 95 \%[\mathrm{CI}] 0.973-0.994$ in multivariate analysis). Our study showed that the dynamic change of TIN could better predict the chemotherapy efficacy and prognosis than the stationary state of TIN after NACT. The reason may be that the dynamic change of TIN partially reflected the response of TME to NACT, which indicated whether pro-tumor inflammation or anti-tumor immune played a major role in TME. For the high difference group patients, the reduction of TIN may reduce the impact on anti-tumor immune response, leading to better immune

Table 3 Clinicopathological Characteristics: Univariate and Multivariate Survival Analyses

\begin{tabular}{|c|c|c|c|c|}
\hline \multirow[t]{3}{*}{ Characteristic } & \multicolumn{4}{|l|}{ DFS } \\
\hline & \multicolumn{2}{|l|}{ Univariate } & \multicolumn{2}{|l|}{ Multivariate } \\
\hline & HR $(95 \% \mathrm{Cl})$ & $P$ & HR $(95 \% \mathrm{Cl})$ & $P$ \\
\hline Age & $1.033(0.995-1.072)$ & 0.007 & $1.031(0.973-1.093)$ & 0.295 \\
\hline Menopause vs no menopause & $1.693(0.672-4.268)$ & 0.257 & $0.927(0.227-3.790)$ & 0.916 \\
\hline Stage (III vs II vs I) & $4.411(1.396-13.938)$ & 0.015 & $3.801(1.163-12.427)$ & 0.027 \\
\hline Molecular type (HR+ vs Her-2 vs TNBC) & $0.926(0.559-1.534)$ & 0.756 & $0.916(0.606-1.384)$ & 0.676 \\
\hline Low TIN after NACT VS High TIN after NACT & $3.415(1.489-7.831)$ & 0.002 & $3.265(1.374-7.758)$ & 0.007 \\
\hline
\end{tabular}

Abbreviation: HR Hormone receptor, Her-2 Human epidermal growth factor receptor-2, TNBC Triple negative breast cancer, DFS Disease free survival, TIN Tumor infiltrating neutrophil, NACT Neoadjuvant chemotherapy 
Table 4 Clinicopathological profiles of the paired patients treated with NACT

\begin{tabular}{|c|c|c|c|c|}
\hline \multirow{2}{*}{\multicolumn{2}{|c|}{ Characteristic }} & \multirow{2}{*}{$\begin{array}{l}\text { Low difference value } \\
N=41\end{array}$} & \multirow{2}{*}{$\begin{array}{l}\text { High difference value } \\
N=48\end{array}$} & \multirow[t]{2}{*}{$P$} \\
\hline & & & & \\
\hline \multicolumn{2}{|c|}{ Age } & $54.73 \pm 11.052$ & $53.06 \pm 11.434$ & 0.636 \\
\hline \multicolumn{2}{|c|}{$<40$} & 3 & 9 & 0.115 \\
\hline \multicolumn{2}{|c|}{$>40$} & 38 & 39 & \\
\hline \multicolumn{2}{|c|}{ No menopause } & 15 & 19 & 0.772 \\
\hline \multicolumn{2}{|c|}{ Menopause } & 26 & 29 & \\
\hline \multicolumn{2}{|l|}{ T1 } & 10 & 6 & 0.394 \\
\hline \multicolumn{2}{|l|}{ T2 } & 22 & 30 & \\
\hline \multicolumn{2}{|l|}{ T3 } & 7 & 7 & \\
\hline \multicolumn{2}{|l|}{ T4 } & 2 & 5 & \\
\hline \multicolumn{2}{|l|}{ No } & 8 & 21 & 0.098 \\
\hline \multicolumn{2}{|l|}{ N1 } & 9 & 8 & \\
\hline \multicolumn{2}{|l|}{ N2 } & 11 & 7 & \\
\hline \multicolumn{2}{|l|}{ N3 } & 13 & 12 & \\
\hline \multicolumn{2}{|c|}{ Stage I } & 4 & 4 & 0.176 \\
\hline \multicolumn{2}{|c|}{ Stage II } & 11 & 22 & \\
\hline \multicolumn{2}{|c|}{ Stage III } & 26 & 22 & \\
\hline \multicolumn{2}{|c|}{$\mathrm{HR}+$} & 28 & 32 & 0.870 \\
\hline \multicolumn{2}{|c|}{ HR- } & 13 & 16 & \\
\hline \multicolumn{2}{|c|}{ Her-2 positive } & 4 & 6 & 0.683 \\
\hline \multicolumn{2}{|c|}{ Her-2 negative } & 37 & 42 & \\
\hline \multicolumn{2}{|c|}{ TNBC vs } & 9 & 10 & 0.898 \\
\hline \multicolumn{2}{|c|}{ No TNBC } & 32 & 38 & \\
\hline \multicolumn{2}{|c|}{ Remission vs } & 22 & 45 & $<0.001$ \\
\hline \multicolumn{2}{|c|}{ No remission } & 19 & 3 & \\
\hline \multicolumn{2}{|c|}{ recurrence } & 12 & 5 & 0.024 \\
\hline \multicolumn{2}{|c|}{ No recurrence } & 29 & 43 & \\
\hline \multirow[t]{4}{*}{ CR } & TEC & 14 & 17 & 0.495 \\
\hline & EC & 8 & 14 & \\
\hline & $\mathrm{EC}$ & 5 & 7 & \\
\hline & TX & 14 & 10 & \\
\hline
\end{tabular}

Abbreviation: HR Hormone receptor, Her-2 Human epidermal growth factor receptor-2, TNBC Triple negative breast cancer, NACT Neoadjuvant chemotherapy, CR Chemical regime, TEC Docetaxel + anthracyclines + cyclophosphamide, EC Anthracyclines + cyclophosphamide, TC Docetaxel + cyclophosphamide, DP Docetaxel + platinum

killing effect conducted by TIL, and favorable chemotherapy efficacy. Therefore, it may serve as a marker for evaluating the curative effect and predicting prognosis in patients with breast cancer patients receiving NACT, and may provide a new insight for immunotherapy of breast cancer in the future.

Some limitations in the present study need to be considered. First, the number of patients enrolled in this study was relatively small. In order to better evaluate and analyze the correlation between TIN and the chemotherapy efficacy and prognosis of breast cancer patients, more patients need to be enrolled in the study from multiple centers to verify the results using a validation cohort and to improve the credibility of the study. Second, our study didn't explore the mechanism of TIN in TME. Therefore, these results still need a prospective, large, multicentered randomized trial to validate.

\section{Conclusion}

TIN after NACT was identified as an independent prognostic factor for breast cancer patients. Furthermore, we revealed that the difference of TIN before and after NACT may become a new, strong, independent prognostic factor for clinical outcome of patients with breast cancer. 


\section{Supplementary Information}

The online version contains supplementary material available at https://doi. org/10.1186/s12885-021-07789-6

Additional file 1: Supplement Figure 1. Chemotherapy regime in low and high difference group.

Additional file 2: Supplement Figure 2. Analysis of patients achieving remission after NACT in high and low TIN groups (a), in low and high difference group (b). TIN = tumor infiltrating neutrophil.

Additional file 3: Supplement Figure 3. Representative microphotographs of CD66b staining. Tumor tissue with no CD66b staining (a). Tumor tissue with weak CD66b staining (b). Tumor tissue with CD66b strong staining (c), (magnification 200x).

Additional file 4: Supplement Figure 4. Representative microphotographs of CD66b staining before and after NACT. CD66b staining before NACT $(a, c)$ and CD66b staining after NACT $(b, d)$. Positive staining was brown and magnification was 200x. The representative staining $a$ and $b$ were from the same patient (low difference group). C and $d$ were from another patient (high difference group).

Additional file 5: Supplement Table 1. Clinicopathological Characteristics: Univariate and Multivariate Survival Analyses.

\section{Abbreviations}

TIN: Tumor infiltrating neutrophil; BCS: Breast-conserving surgery; HR: Hormone receptor; Her-2: Human epidermal growth factor receptor-2; TNBC: Triple negative breast cancer; DFS: Disease-free survival; NACT: Neoadjuvant chemotherapy; PCR: Pathological complete response; CR: Chemical regime; TEC: Docetaxel + anthracyclines + cyclophosphamide; EC: Anthracyclines + cyclophosphamide; TC: Docetaxel + cyclophosphamide; DP: Docetaxel + platinum; TME: Tumor microenvironment

\section{Acknowledgements}

None.

\section{Authors' contributions}

All authors made substantial contributions to one or more of the following: the study conception and design: SK G, YP F and HW Z; provision of study materials or patients: SK G and HW Z; collection and assembly of data: SM F, SH M and SK G; data analysis and interpretation: SK G, YP F, SH M and HW Z. SK G and SM F drafted the article. YP F and HW Z contributed to revising the article critically for important intellectual content. All authors read and approved the final manuscript.

\section{Funding}

This research was supported by the National Natural Science Foundation of China (grant numbers 81902993, the funder was Yi-Peng Fu). Yi-Peng Fu made substantial contributions in study conception and design, data analysis and interpretation and revising the article.

\section{Availability of data and materials}

The dataset of the current study was available from the corresponding author on reasonable request.

\section{Ethics approval and consent to participate}

This study was approved by the Institutional Review Board of Zhongshan Hospital affiliated to Fudan University. Written informed consent was obtained from all participants included in the study.

\section{Consent for publication}

Not applicable.

\section{Competing interests}

The authors declared no conflicts of interests.

\section{Author details}

${ }^{1}$ Department of General Surgery, Xuhui District Central Hospital of Shanghai, Shanghai 200031, China. ${ }^{2}$ Department of Breast Surgeon, The Obstetrics \& Gynecology Hospital of Fudan University, Shanghai 200011, China.
${ }^{3}$ Department of General Surgery, Zhongshan Hospital, Fudan University, Shanghai 200032, China.

Received: 27 June 2020 Accepted: 4 January 2021

Published online: 14 January 2021

\section{References}

1. DeSantis C, Ma J, Bryan L, Jemal A. Breast cancer statistics, 2013. CA Cancer J Clin. 2014;64(1):52-62.

2. Chen W, Zheng R, Baade PD, Zhang S, Zeng $H$, Bray F, Jemal A, Yu XQ, He J. Cancer statistics in China, 2015. CA Cancer J Clin. 2016:66(2):115-32.

3. Szollar A, Ujhelyi M, Polgar C, Olah E, Pukancsik D, Rubovszky G, Udvarhelyi N, Kovacs T, Savolt A, Kenessey I, et al. A long-term retrospective comparative study of the oncological outcomes of 598 very young $(</=35$ years) and young (36-45 years) breast cancer patients. Eur J Surg Oncol. 2019:45(11):2009-15

4. Mantovani A, Allavena P, Sica A, Balkwill F. Cancer-related inflammation. Nature. 2008:454(7203):436-44

5. Vesely MD, Schreiber RD. Cancer immunoediting: antigens, mechanisms, and implications to cancer immunotherapy. Ann N Y Acad Sci. 2013:1284:15 .

6. Dunn GP, Bruce AT, Ikeda H, Old LJ, Schreiber RD. Cancer immunoediting: from immunosurveillance to tumor escape. Nat Immunol. 2002;3(11):991-8.

7. Schreiber RD, Old LJ, Smyth MJ. Cancer immunoediting: integrating immunity's roles in cancer suppression and promotion. Science. 2011; 331(6024):1565-70.

8. Hanahan D, Weinberg RA. Hallmarks of cancer: the next generation. Cell. 2011;144(5):646-74

9. Shaul ME, Fridlender ZG. Cancer-related circulating and tumor-associated neutrophils - subtypes, sources and function. FEBS J. 2018;285(23):4316-42

10. Ou X, Tang Y, Hua S. Immunological approaches towards Cancer and inflammation: a cross talk. Front Immunol. 2018;9:563.

11. Liang $\mathbf{W}$, Ferrara $\mathrm{N}$. The complex role of neutrophils in tumor angiogenesis and metastasis. Cancer Immunol Res. 2016;4(2):83-91.

12. Masucci MT, Minopoli M, Carriero MV. Tumor Associated Neutrophils. Their Role in Tumorigenesis, Metastasis, Prognosis and Therapy. Front Oncol. 2019:9:1146

13. Galdiero MR, Bianchi P, Grizzi F, Di Caro G, Basso G, Ponzetta A, Bonavita E, Barbagallo M, Tartari S, Polentarutti N, et al. Occurrence and significance of tumor-associated neutrophils in patients with colorectal cancer. Int J Cancer. 2016;139(2):446-56.

14. Donskov F, von der Maase $\mathrm{H}$. Impact of immune parameters on long-term survival in metastatic renal cell carcinoma. J Clin Oncol. 2006;24(13):19972005.

15. Trellakis S, Farjah H, Bruderek K, Dumitru CA, Hoffmann TK, Lang S, Brandau S. Peripheral blood neutrophil granulocytes from patients with head and neck squamous cell carcinoma functionally differ from their counterparts in healthy donors. Int J Immunopathol Pharmacol. 2011:24(3):683-93.

16. Wang Y, Chen J, Yang L, Li J, Wu W, Huang M, Lin L, Su S. Tumor-contacted neutrophils promote metastasis by a CD90-TIMP-1 Juxtacrine-paracrine loop. Clin Cancer Res. 2019;25(6):1957-69.

17. Shaul ME, Fridlender ZG. Neutrophils as active regulators of the immune system in the tumor microenvironment. J Leukoc Biol. 2017;102(2):343-9.

18. Jensen HK, Donskov F, Marcussen N, Nordsmark M, Lundbeck F, von der Maase $\mathrm{H}$. Presence of intratumoral neutrophils is an independent prognostic factor in localized renal cell carcinoma. J Clin Oncol. 2009;27(28): 4709-17.

19. Zhang H, Liu H, Shen Z, Lin C, Wang X, Qin J, Qin X, Xu J, Sun Y. Tumorinfiltrating neutrophils is prognostic and predictive for postoperative adjuvant chemotherapy benefit in patients with gastric Cancer. Ann Surg. 2018;267(2):311-8.

20. Shah TJ, Leik CE, Walsh SW. Neutrophil infiltration and systemic vascular inflammation in obese women. Reprod Sci. 2010;17(2):116-24.

21. Kawahara T, Furuya K, Nakamura M, Sakamaki K, Osaka K, Ito H, Ito Y, Izumi K, Ohtake S, Miyoshi Y, et al. Neutrophil-to-lymphocyte ratio is a prognostic marker in bladder cancer patients after radical cystectomy. BMC Cancer. 2016;16:185

22. Litiere S, Collette S, de Vries EG, Seymour L, Bogaerts J. RECIST - learning from the past to build the future. Nat Rev Clin Oncol. 2017;14(3):187-92.

23. Fisher B, Bryant J, Wolmark N, Mamounas E, Brown A, Fisher ER, Wickerham DL, Begovic M, DeCillis A, Robidoux A, et al. Effect of preoperative 
chemotherapy on the outcome of women with operable breast cancer. J Clin Oncol. 1998;16(8):2672-85.

24. Toi M, Nakamura S, Kuroi K, Iwata H, Ohno S, Masuda N, Kusama M, Yamazaki K, Hisamatsu K, Sato Y, et al. Phase II study of preoperative sequential FEC and docetaxel predicts of pathological response and disease free survival. Breast Cancer Res Treat. 2008;110(3):531-9.

25. Jia JB, Wang WQ, Sun HC, Zhu XD, Liu L, Zhuang PY, Zhang JB, Zhang W, Xu HX, Kong LQ, et al. High expression of macrophage colony-stimulating factor-1 receptor in peritumoral liver tissue is associated with poor outcome in hepatocellular carcinoma after curative resection. Oncologist. 2010;15(7): $732-43$

26. Shah TJ, Walsh SW. Activation of NF-kappaB and expression of COX-2 in association with neutrophil infiltration in systemic vascular tissue of women with preeclampsia. Am J Obstet Gynecol. 2007;196(1):48 e41-8.

27. Adams S, Gray RJ, Demaria S, Goldstein L, Perez EA, Shulman LN, Martino S, Wang $\mathrm{M}$, Jones VE, Saphner TJ, et al. Prognostic value of tumor-infiltrating lymphocytes in triple-negative breast cancers from two phase III randomized adjuvant breast cancer trials: ECOG 2197 and ECOG 1199. J Clin Oncol. 2014;32(27):2959-66.

28. Denkert C, von Minckwitz G, Darb-Esfahani S, Lederer B, Heppner BI, Weber KE, Budczies J, Huober J, Klauschen F, Furlanetto J, et al. Tumour-infiltrating lymphocytes and prognosis in different subtypes of breast cancer: a pooled analysis of 3771 patients treated with neoadjuvant therapy. Lancet Oncol. 2018;19(1):40-50.

29. West NR, Kost SE, Martin SD, Milne K, Deleeuw RJ, Nelson BH, Watson PH. Tumour-infiltrating FOXP3(+) lymphocytes are associated with cytotoxic immune responses and good clinical outcome in oestrogen receptornegative breast cancer. Br J Cancer. 2013;108(1):155-62.

30. Mahmoud SM, Paish EC, Powe DG, Macmillan RD, Grainge MJ, Lee AH, Ellis IO, Green AR. Tumor-infiltrating CD8+ lymphocytes predict clinical outcome in breast cancer. J Clin Oncol. 2011;29(15):1949-55.

31. Hida Al, Watanabe T, Sagara Y, Kashiwaba M, Sagara Y, Aogi K, Ohi Y, Tanimoto A. Diffuse distribution of tumor-infiltrating lymphocytes is a marker for better prognosis and chemotherapeutic effect in triple-negative breast cancer. Breast Cancer Res Treat. 2019:178(2):283-94.

32. Gregory AD, Houghton AM. Tumor-associated neutrophils: new targets for cancer therapy. Cancer Res. 2011;71(7):2411-6.

33. Wang J, Bo X, Suo T, Liu H, Ni X, Shen S, Li M, Xu J, Liu H, Wang Y. Tumorinfiltrating neutrophils predict prognosis and adjuvant chemotherapeutic benefit in patients with biliary cancer. Cancer Sci. 2018;109(7):2266-74.

34. Wculek SK, Malanchi I. Neutrophils support lung colonization of metastasisinitiating breast cancer cells. Nature. 2015;528(7582):413-7.

\section{Publisher's Note}

Springer Nature remains neutral with regard to jurisdictional claims in published maps and institutional affiliations.

Ready to submit your research? Choose BMC and benefit from:

- fast, convenient online submission

- thorough peer review by experienced researchers in your field

- rapid publication on acceptance

- support for research data, including large and complex data types

- gold Open Access which fosters wider collaboration and increased citations

- maximum visibility for your research: over $100 \mathrm{M}$ website views per year

At BMC, research is always in progress.

Learn more biomedcentral.com/submissions 